\title{
Substantiation of fuzzy logic algorithms for control problems of a geotechnical systems
}

\author{
Anton Slashchov, ${ }^{1,}$, and Oleksii Yalanskyi ${ }^{2}$ \\ ${ }^{1}$ Institute of Geotechnical Mechanics named by N. Poljakov of National Academy of Sciences of \\ Ukraine, 49005, Dnipro, Simferopolska Str., 2a, Ukraine \\ ${ }^{2}$ National Technical University Dnipro Polytechnic, 49005, Dnipro, Dmytra Yavornytskoho Ave., 19, \\ Ukraine
}

\begin{abstract}
The article presents results of study of intelligent fuzzy logic algorithms developed on the basis of fuzzy logic methods for information system of the mine safety system. In order to prevent emergency situations caused by the lost geotechnical system stability due to the uncertain behavior of the rock mass, a new fuzzy controller was designed which could generate an additional control signal. For the fuzzy controller, methods of data fuzziness, inference and de-fuzziness were validate, and linguistic rules were designed in order to control parameters of the geotechnical system. With the help of the Cauchy problem solved by Runge-Kutta method of the 4th order, designed a software model of the proposed system which simulated the system operation. The model has proved operability and static stability of the developed algorithms. Output signal of the fuzzy controller can be used as information for estimating risk for geotechnical systems, preventing possible emergency situations and, consequently, can improve job safety in the mines.
\end{abstract}

\section{Introduction}

The control systems based on fuzzy logic methods [1,2] are among the most promising for practical application. The technical implementation of control systems embodying intelligent methods and algorithms is based on the use of computers and microprocessor tools. Fuzzy control systems are especially useful when processes in a controlled system are too complex to analyze using traditional quantitative methods, or when incoming informative parameters cannot be reliably interpreted due to the impossibility of establishing accurate criteria for evaluating the source data. These uncertainties often do not allow monitoring and predicting of processes in geotechnical systems.

Geotechnical systems are monitored for safety of mining operations under partial uncertainty of the rock massif behavior under load, irregularity and randomness of the distribution in space of the properties and structural features of rocks, variety characteristics of roadways supporting and other poorly predictable factors [3]. Unfortunately, in the mining and processing industries, the approaches to the development of management

\footnotetext{
*Corresponding author: AISlashchov@nas.gov.ua
} 
systems are still deeply conservative, which is connected with the need to ensure safe and trouble-free operation of technical systems in the difficult conditions of the development of deposits at great depths, danger of sudden emissions of rocks and gases. The applied classical control systems are effective for controlling strictly determinate objects and do not work well in conditions of incomplete and/or distorted information. Therefore, the construction in addition to the already existing safety systems of new intelligent algorithms is relevant and in demand in production.

\section{Methods}

Research methods: mathematical theory of fuzzy sets and fuzzy logic; methods for implementing fuzzy logic algorithms (fuzzification, inference and defuzzification) for converting input and output signals, creating expert rules and ensuring the functioning of the fuzzy controller; methods of organizing computational processes and building software models of information systems for constructing a model and analyzing the fuzzy controller process; simulation of fuzzy controller operation.

\section{Results and discussion}

At the Institute of Geotechnical Mechanics named by N. Poljakov of National Academy of Sciences of Ukraine, work is underway to improve mining safety systems [3,4] involving geomechanical models and methods for assessing scenarios for the development of situations. Maintaining the equilibrium state of the geotechnical system, as an object of management, provides for the control of the system parameters values from the equilibrium point and the rate of change of this deviation. On the basis of this data, control actions and personnel warning signals are generated. When a signal arrives at the dispatcher console or at the input of an automated control system for an object, a recommendation is drawn up for the use of technical and technological measures that contribute to the normalization of the system. For the analysis of incoming data on the state of rocks and roadways, as well as the formation of a timely response to changes in the parameters of the "support-rock massif" geotechnical system, algorithms based on fuzzy logic methods were developed.

For the two-coordinate fuzzy controller, it is proposed to use the input parameters "Deviation" ("Del") and "Derivative deviation" ("dDel/ dt", Fig. 1). To obtain the value of the derivative, a differentiating element was introduced into the fuzzy controller structure. At the output of the controller "Output", a control signal is generated, which is one of the control criteria in the system for ensuring the assessment of the state of the geological environment, including mine roadways and means for their maintenance. It can also be used as a correction signal to control signals, which generated by classical control systems. For example, the fuzzy controller signal can be applied to directly correct the value of the penetration rate setting for a mining combine to prevent rocks fall and other dangerous manifestations of rock pressure.

During fuzzy controller operation, fuzzification (translation of the exact values of input signals into the value of logical-linguistic variables), inference (formation of rules by experts and their conversion into control law) and defuzzification (conversion of a fuzzy set into a clear number) are performed. The control law can be implemented by software and hardware if the system is configured to generate and transmit danger signals, as well as to initiate the creation of guidelines that regulate the implementation of technical measures.

The fuzzification process determines the values of all membership functions for the current input values of the deviation arguments and the derivative of the deviation. For the accepted distribution of intervals and triangular membership functions at any time in the 
operating area for only two functions, their values will differ from zero and one. In the general case, the integral value of all membership functions in a single input variable, regardless of the current value of the argument, is equal to one, which is one of the advantages and, in this case, determined the use of triangular activation functions.

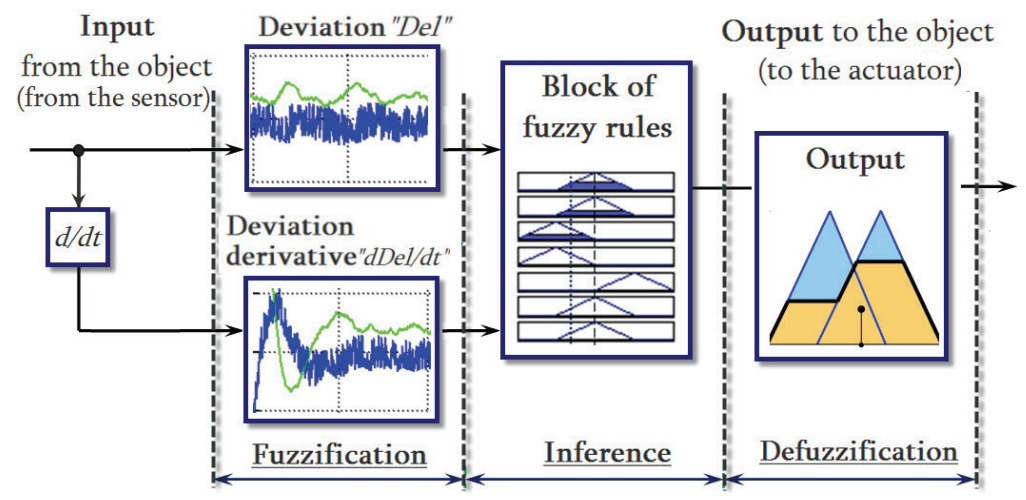

Fig. 1. The signal processing circuit in fuzzy controller.

As a result of fuzzification, we have, in general, four non-zero values of the membership functions (two values for each of the two input axes), which must be processed using the fuzzy logic mathematical apparatus. In the developed device, the maximum aggregation of these values is performed (a graphical interpretation of individual stages of inference is shown in Figure 2).

a)

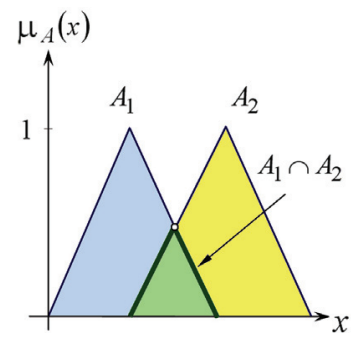

c)
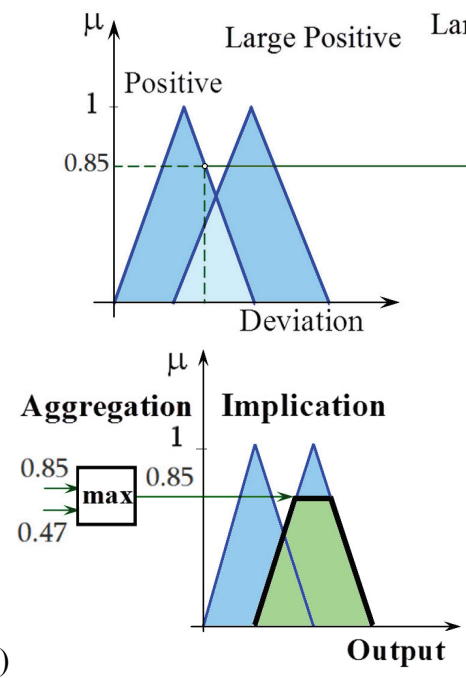

b)

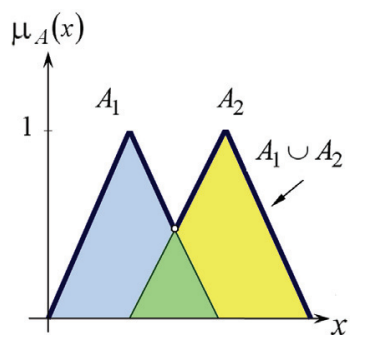

Large Negativ
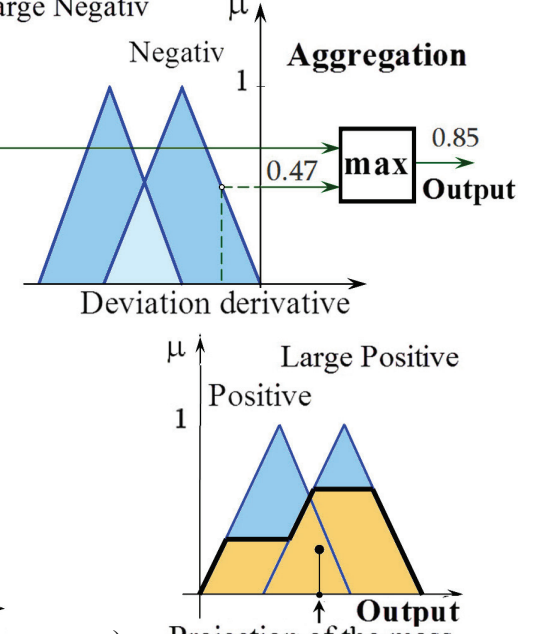

e) Projection of the mass

Fig. 2. Methods of calculations used in fuzzy controller: intersection (a) and union (b) of fuzzy sets, aggregation processes (c) and implication (d), defuzzification of the result of calculations (e). 
The calculation of inference stages parameters (intersection, union, aggregation and implication of fuzzy sets) is carried out using known analytical relations $[1,5]$. The controller also implemented a minimum ("Mamdani") implication for defining output fuzzy sets for each of the active rules. The presentation of the data processing methods adopted in the controller is given in Table 1 .

Table 1. Parameters of the fuzzy controller under study to prevent emergency situations caused by the loss of equilibrium of the geotechnical system.

\begin{tabular}{|l|l|l|c|l|}
\hline № & \multicolumn{1}{|c|}{ Parameter } & Value & Figure & \multicolumn{1}{c|}{ Analytic Expression } \\
\hline 1 & $\begin{array}{l}\text { Logical multiplicatio } \\
\text { method (fuzzy intersection) }\end{array}$ & Min & Fig. 2, a & $\mu_{A_{1} \cap A_{2}}(x)=\min \left\{\mu_{A_{1}}(x), \mu_{A_{2}}(x)\right\}$ \\
\hline 2 & $\begin{array}{l}\text { Logical addition method } \\
\text { (fuzzy union) }\end{array}$ & Max & Fig. 2, b & $\mu_{A_{1} \cup A_{2}}(x)=\max \left\{\mu_{A_{1}}(x), \mu_{A_{2}}(x)\right\}$ \\
\hline 3 & Aggregation method & Max & Fig. 2, c & $\begin{array}{l}E_{i}(x)=\max \left\{E_{1, i}\left(x_{1}\right), \ldots, E_{m, i}\left(x_{m}\right)\right\} \\
=\max _{k=1}\left\{E_{k, i}\left(x_{k}\right)\right\}, i \subset\{1, \ldots, n\}\end{array}$ \\
\hline 4 & Implication method & Min & Fig. 2, d & $\mu_{B_{i}^{*}}(c)=\min \left\{E_{i}(x), \mu_{B_{i}}(c)\right\}$ \\
\hline 5 & Defuzzification method & Gravita- & Fig. 2, e & $X_{S}=\frac{\int c \cdot \mu(c) d c}{\int \mu(c) d c}$ \\
\hline
\end{tabular}

The notation in the table: $A, A_{1}, A_{2}$ are fuzzy sets; $\mu_{A}(x)$ is the degree to which the $x$ value belongs to the fuzzy set; $A ; E_{i}(x)$ is the degree of belonging with which the elementary expression "If"-parts of each active fuzzy rule is true, and this $i \in\{1, \ldots, n\}$ is the sequence number of the fuzzy rule, the total number $n$, and $m$ is the total number of elementary expressions for one rule; $B_{i}$ is the fuzzi-set defined for implication for each $i$-th rule, depending on the degree of belonging $E_{i}(x)$ with which it is executed; $X_{S}$ is the projection of the center of mass of the membership function of the output value on the $x$-axis.

For each of the input variables, the $x$-axis within the operating area is divided into five intervals, conventionally designated according to established terminology: a large negative value of the argument " $L N$ " (Large Negative), a negative value of the argument " $N$ " (Negative), a zero value of the argument " $Z$ ' (Zero), positive value " $P$ " (Positive), large positive value of the argument " $L P$ " (Large Positive). As a result of fuzzification, we have, in general, four non-zero values of the membership functions (two values for each of the two input axes), which must be processed using the fuzzy logic mathematical apparatus.

To prevent emergencies caused by the loss of equilibrium of the geotechnical system, the following methods for processing input signals are implemented in the inference unit of the fuzzy controller developed:

- method of logical multiplication (intersection of fuzzy-sets):

$$
\mu_{A_{1} \cap A_{2}}(x)=\min \left\{\mu_{A_{1}}(x), \mu_{A_{2}}(x)\right\},
$$

where $A, A_{1}, A_{2}$ are fuzzy sets, $\mu_{A}(x)$ is the degree to which $x$ is a fuzzy set $A$;

- method of logical addition (union of fuzzy-sets):

$$
\mu_{A_{1} \cup A_{2}}(x)=\max \left\{\mu_{A_{1}}(x), \mu_{A_{2}}(x)\right\} ;
$$

- aggregation method (association according to the rule): 


$$
E_{i}(x)=\max \left\{E_{1, i}\left(x_{1}\right), \ldots, E_{m, i}\left(x_{m}\right)\right\}=\max _{k=1}^{m}\left\{E_{k, i}\left(x_{k}\right)\right\}, i \subset\{1, \ldots, n\},
$$

where $E_{i}(x)$ is the degree of belonging with which the elementary expression "If"-part of each active fuzzy rule is true, $i \in\{1, \ldots, n\}$ is the ordinal number of the fuzzy rule, the total number $n$, and $m$ is the total number of elementary expressions for one rule;

- implication method:

$$
\mu_{B_{i}^{*}}(c)=\min \left\{E_{i}(x), \mu_{B_{i}}(c)\right\}
$$

where $B_{i}$ is the a fuzzy-set defined during implication for each $i$-th rule, depending on the degree of belonging $E_{i}(x)$, with which it is executed;

- defuzzification method (gravitational)

$$
X_{S}=\frac{\int c \cdot \mu(c) d c}{\int \mu(c) d c}
$$

where $X_{S}$ - the projection of the center of mass of the membership function of the output value on the $x$-axis.

The linguistic rules for the functioning of the controller are formulated by an expert based on his knowledge and skills of managing an object. For the fuzzy controller to prevent emergencies caused by the loss of the equilibrium state of the geotechnical system "support-rock massif", the following rules for generating a compensating effect are proposed:

- if "Deviation" is increasing rapidly

$$
\text { If (Del is LP) and }((d D e l / d t) \text { is LP) then (Output is LN); }
$$

- if "Deviation" is large negative, and it increases

$$
\text { If (Del is LN) and }((d D e l / d t) \text { is LN) then (Output is LP); }
$$

- if "Deviation" is big positive, and "Derivative deviation" is big negative

$$
\text { If (Del is LP) and ((dDel / dt) is LN) then (Output is Z); }
$$

- if "Deviation" is positive and "Derivative deviation" is also positive

$$
\text { If (Del is } P) \text { and }((d D e l / d t) \text { is } P) \text { then (Output is } N) \text {; }
$$

- if "Deviation" is negative and "Derivative deviation" is also negative

$$
\text { If }(\text { Del is } N) \text { and }((d \mathrm{Del} / d t) \text { is } N) \text { then (Output is } P) \text {. }
$$

Under some conditions, it is proposed to use two rules, each with weighting coefficients of 0.5. So, if the "Deviation" value is large positive $L P$, and the speed is positive $P$, i.e. the deviation increases, although not too quickly, the controller output is calculated as the result of the composition of two rules that form a large negative $L N$ and negative $N$ values of the corrective action:

$$
\begin{aligned}
& \text { If }(\text { Del is LP) and }((d \mathrm{Del} / d t) \text { is P }) \text { then }(\text { Output is LN), } k=0.5 ; \\
& \text { If }(\text { Del is LP) and }((d D e l / d t) \text { is P) then }(\text { Output is } N), k=0.5,
\end{aligned}
$$


where $k$ - the weighting factor for the rule (the sum of the weighting factors for all the rules within one cell of the table of the rules we have formulated is one, Table 1).

Table 1. The linguistic rules of the developed controller.

\begin{tabular}{|c|c|c|c|c|c|c|}
\hline \multirow{2}{*}{\multicolumn{2}{|c|}{$\begin{array}{c}\text { Input Coordinates and Split } \\
\text { Ranges }\end{array}$}} & \multicolumn{5}{|c|}{ Input value "Deviation" ( $\mathrm{Del})$} \\
\hline & & $L N$ & $N$ & $Z$ & $P$ & $L P$ \\
\hline \multirow{5}{*}{$\begin{array}{l}\text { Input value } \\
\text { "Derivative } \\
\text { deviation" } \\
(d D e l / d t)\end{array}$} & $L N$ & $L P$ & $\begin{array}{l}0.5 \mathrm{LP} \\
0.5 P\end{array}$ & $P$ & $\begin{array}{l}0.5 P \\
0.5 Z\end{array}$ & $Z$ \\
\hline & $N$ & $\begin{array}{c}0.5 \mathrm{LP} \\
0.5 P\end{array}$ & $P$ & $\begin{array}{l}0.5 P \\
0.5 Z\end{array}$ & $Z$ & $\begin{array}{l}0.5 \mathrm{Z} \\
0.5 \mathrm{~N}\end{array}$ \\
\hline & $Z$ & $P$ & $\begin{array}{l}0.5 P \\
0.5 Z\end{array}$ & $Z$ & $\begin{array}{l}0.5 \mathrm{Z} \\
0.5 \mathrm{~N}\end{array}$ & $N$ \\
\hline & $P$ & $\begin{array}{l}0.5 P \\
0.5 Z\end{array}$ & $Z$ & $\begin{array}{l}0.5 \mathrm{Z} \\
0.5 \mathrm{~N}\end{array}$ & $N$ & $\begin{array}{l}0.5 \mathrm{~N} \\
0.5 \mathrm{LN}\end{array}$ \\
\hline & $L P$ & $Z$ & $\begin{array}{l}0.5 \mathrm{Z} \\
0.5 \mathrm{~N}\end{array}$ & $N$ & $\begin{array}{l}0.5 \mathrm{~N} \\
0.5 \mathrm{LN}\end{array}$ & $L N$ \\
\hline
\end{tabular}

To test the effectiveness of the solutions used, developed a program model of the proposed system and simulated its work with solving the Cauchy problem using the 4th order Runge-Kutta method (Fig. 3). The control object was represented by aperiodic and oscillatory links with different values of the coefficients of the proportional part and time constants in the terms containing the Laplace operator as a multiplier. It has been established that in all cases studied, the use of a fuzzy controller improved the quality of control: in particular, the overshoot during system start-up and with a sharp increase in load decreased, the static stability of the system improved, which, in turn, reduces the probability of an accident caused by a sudden loss of stability of the control object.

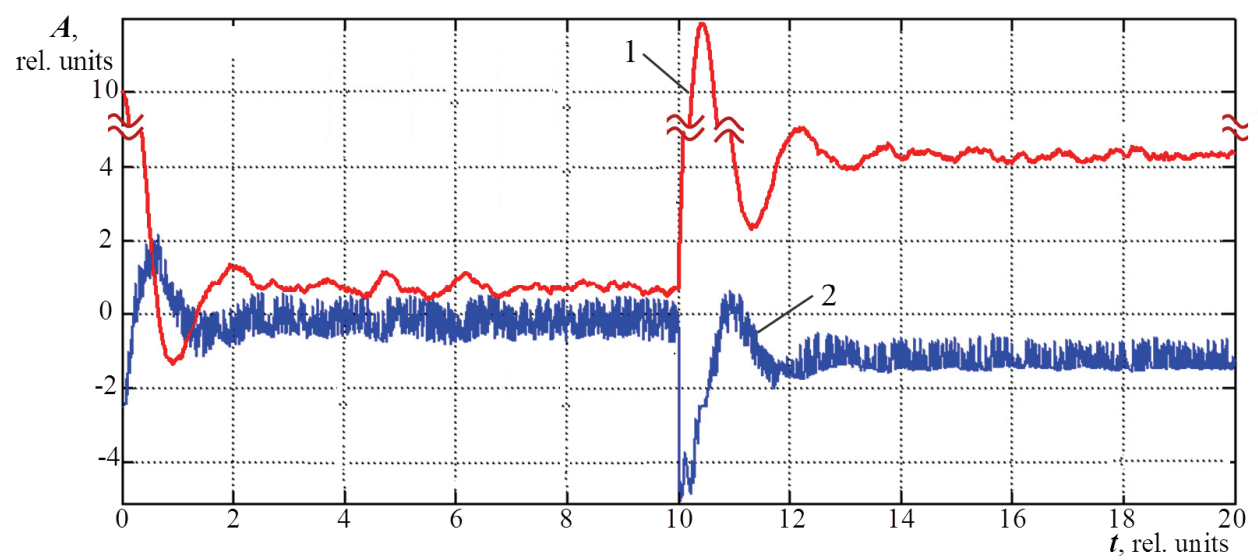

Fig. 3. Modeling fuzzy regulator: 1 - the output signal of the conventional regulator; 2 - correction signal of the fuzzy regulator "Output".

Comparison of the maximum amplitudes showed a low value of the overshoot, which in geotechnical systems can be assessed positively, since the loss of stability as a result of an increase in dynamic loads can lead not only to breakdowns and destruction of the system itself, but also to human victims. The delay time of the response of the control system to external influences and load changes is reduced. This was made possible not only by taking into account the level of deviation of the output value from the target, but by controlling the rate of change of this deviation, including when the absolute value of the deviation in the module itself is insignificant or equal to zero, that is, it is not a sign of a pre-emergency 
situation or a sign of the beginning of the process loss of stability of the geotechnical system.

The output signal of the developed fuzzy controller is used as information to assess the degree of danger of the geotechnical system and prevent possible emergencies, which will increase the safety and productivity of miners.

\section{Conclusions}

For the problem of geotechnical systems monitoring under partial uncertainty of input data and emergency prevention, a fuzzy controller has been developed and tested. Fuzzy controller provides, based on fuzzy logic methods and algorithms, analysis of input data for monitoring the stress-strain state of rocks. The methods of fuzzification, inference and defuzzification of data are justified, linguistic rules to control the parameters of the geotechnical system are developed. A software model of the proposed system was developed. The fuzzy controller is modeled using the 4th order Runge-Kutta method for solving the Cauchy problem. The simulation showed the performance of fuzzy controller algorithms and an increase in the static stability of the controlled system.

The process management process in the geotechnical system can be carried out by software by generating and transmitting danger signals, as well as initiating the creation of guidelines for the implementation of technical measures, which ultimately will ensure the effectiveness of warning personnel about possible emergency situations and increase occupational safety on mining enterprises.

\section{References}

1. L.A. Zadeh, Fuzzy Sets. Fuzzy Logic. Fuzzy Systems (World Scientific Press, 1996)

2. R.V. Demicco, G.J. Klir. Fuzzy Logic in Geology (Academic Press, 2003)

3. Bulat, A.F., Slashchov, I.M., Slashchova, O.A. (2017). Evaluation methods of interconnected geomechanical and gas dynamic processes in the rock massif for the systems of working medium control in the mines, Geotekhnicheskaya Mekhanika, 59, 34-44

4. Slashchov, A.I. (2016). Justification of the parameters of the information system assuring the underground mining safety, Naukovyi Visnyk Natsionalnoho Hirnychoho Universytetu, 1, 77-85

5. Kalashnikov, V.I., Palis, F., Lozynskyy, O.Yu. (2000). Osnovy teoriyi fazy-lohiky ta fazy-rehulyuvannya. Donetsk-Magdeburg-Lviv 